\title{
Peripherally Inserted Central Catheter Use in the Hospitalized Patient: Is There a Role for the Hospitalist?
}

Adam S. Akers, mD Lakshmipathi Chelluri, MD, MPH

\begin{abstract}
Department of Critical Care Medicine, University of Pittsburgh Medical Center, Pittsburgh, Pennsylvania.
\end{abstract}
University of Pittsburgh IRB\#: PRO08020126.

Disclosure: Nothing to report.

BACKGROUND: Peripherally-inserted central venous catheters (PICCs) are frequently used in hospitals for central intravenous access. These catheters may offer advantages over traditional central catheters with respect to ease of placement and decreased complication rates. However, hospital physicians have not traditionally been trained to place PICCs.

METHODS: We trained 3 of 5 hospitalists to place PICCs in our small university-affiliated community hospital as we converted from a house physician model to a hospitalist model for inpatient care. We then looked retrospectively at the rates of all PICC and other central catheter placements as well as the number of femoral and nonfemoral catheter days for the 18-month period prior to and after the inception of the hospitalist program.

RESULTS: Comparing the periods prior to and after the inception of the hospitalist program, the total number of central catheter placements doubled and the PICC rate rose from $20 \%$ to $80 \%$ of all central catheters. The rate of femoral and subclavian catheter placements decreased by approximately $50 \%$ and the rate of internal jugular catheter placement was roughly unchanged. There was also a fall in the number of femoral catheter days and a great increase in the number of total nonfemoral catheter days. The rate of catheter-related bacteremia remained low and did not appear to increase.

CONCLUSIONS: PICCs may be a safe and easy alternative to centrally placed catheters for the hospital physician attempting to secure central intravenous access and may lead to a decrease in the need for more risky central venous catheter (CVC) insertions. Journal of Hospital Medicine 2009;4:E1-E4. @ 2009 Society of Hospital Medicine.

KEYWORDS: catheterization, central venous, infection control, hospitalists.

Peripherally inserted central catheters (PICCs) are being used with greater frequency than ever before for intravenous access in hospitals, and PICCs may offer advantages in safety over traditional central venous catheters (CVCs). Despite these potential advantages, a large number of CVCs are still being placed. In a recent 1-day survey of 6 large urban teaching hospitals, $29 \%$ of all patients had a CVC in place $(59.3 \%$ of intensive care unit [ICU] patients and $23.7 \%$ of non-ICU patients). ${ }^{1}$ Most catheters were inserted in the subclavian $(55 \%)$ or jugular $(22 \%)$ veins, with femoral $(6 \%)$ and peripheral $(15 \%)$ sites less commonly used. Even in the non-ICU setting, only $20 \%$ of all central catheters were PICCs.

PICCs may offer advantages over centrally-inserted intravenous catheters, such as the reduced risks of pneumothorax, ${ }^{2}$ arterial puncture, uncontrolled bleeding of large central veins, central line-associated bloodstream infections (CLAB) ${ }^{3,4}$ and lower cost. ${ }^{5}$ In addition, central venous pressure monitoring can now be performed with the larger-bore PICCs. ${ }^{6}$

The low risk of mechanical complications for PICC insertion has been well documented. ${ }^{7,8}$ In contrast, femoral or retroperitoneal hematoma occurs in up to $1.3 \%$ of cases following femoral catheter insertion, ${ }^{9}$ and pneumothorax occurs in $1.5 \%$ to $2.3 \%$ of subclavian catheter insertions. ${ }^{10}$
However, there are only limited data to suggest that the risk of PICC-related bacteremia is lower than that of centrallyplaced catheters. ${ }^{11,12}$

The benefit of PICCs over centrally-placed catheters in terms of venous thromboembolism (VTE) is also not as easy to show, and in fact the rate may be greater in PICCs. The reported incidence of PICC-related VTE has been between $0.3 \%$ and $56.0 \%$, and the wide variation in rates is likely related to the method of diagnosis. ${ }^{13-15}$ It is likely that most patients with PICC-related VTE are asymptomatic, and that its incidence is underestimated. ${ }^{16}$

In many hospitals PICCs are placed by a certified nurse, or by an interventional radiologist if the nurse is unsuccessful. ${ }^{17}$ There are few reports of PICCs being placed by nonradiology physicians. In one report of 894 patients referred to a critical care specialist for PICC insertion, venous access was achieved $100 \%$ of the time, there were no referrals to interventional radiology, and there were no incidents of pneumothorax or bleeding. ${ }^{8}$ In a university-affiliated community hospital, we carried out a retrospective review of our experience with training hospital physicians to place PICCs.

\section{Methods}

In July 2006 our community hospital, which is affiliated with the University of Pittsburgh Medical Center, instituted 


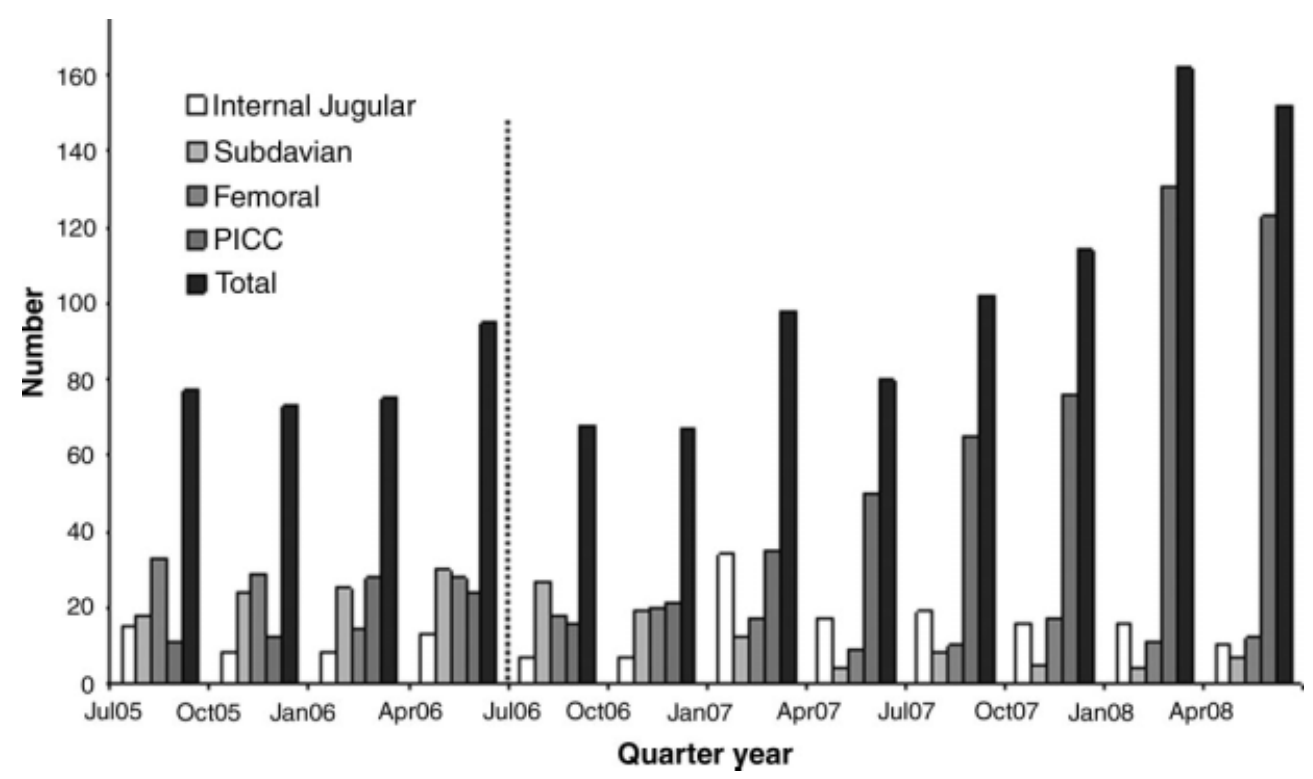

FIGURE 1. Central venous catheter insertion rates by quarter year. The dotted vertical line signifies the beginning of the hospitalist program.

a hospitalist program. Prior to the hospitalist program, 1 house physician was available to place PICCs in the antecubital vein without the aid of ultrasound, and there was no PICC-certified nurse in the hospital. An interventional radiologist was available to place PICCs that could not be placed by the house physician. After July 2006 under the hospitalist service, 3 of the 5 physicians were trained to place PICCs in the deep veins of the arm with the use of ultrasound guidance.

Training included 1 day with the PICC training nurse at the tertiary hospital, followed by supervised placements in the community hospital until proficiency was obtained. Proficiency was relative and cumulative. Approximately 3 supervised procedures were necessary before the physician was able to place PICCs by him or herself. All PICCs were placed using 5 barrier precautions, with chlorhexidine cleansing, and with a "time-out" prior to the procedure.

Retrospective hospital data for central catheter placement were examined for the 18 months prior to and following the start of the hospitalist program. These data were collected routinely by the hospital infection control nurse for purposes of quality improvement and patient safety. The data included central catheters placed by all physicians in the hospital; however, the vast majority of these were placed by the hospitalists. The catheters were placed throughout the hospital, both on the medical floors, cardiac step-down unit, and the ICU. Information regarding the number of central catheters placed and the specific type of catheter (subclavian, jugular, femoral, or PICC) was available from July 2005 through December 2007. Also available from January 2005 were the numbers of femoral and nonfemoral catheter days (number of catheters multiplied by number of days in place) and the central catheter-associated bacteremia rates (number per 1000 catheter days) for femoral and nonfemoral catheters. The Centers for Disease Control and Prevention (CDC) definition of central line-associated bacteremia was used, which is any documented bloodstream infection within 48 hours of the presence of a CVC in the absence of an alternate source of infection. Data for other complications such as pneumothorax and major bleeding were not consistently recorded.

\section{Results}

Figure 1 shows the number of internal jugular, subclavian, femoral, PICC, and total catheter placements from July 2005 through December 2007. The data are grouped into 3 -month increments for visual convenience. Comparing the periods before and after the inception of the hospitalist PICC service (Figure 1, dotted vertical line), the rate of PICC placements rose 4 -fold and the rate of total catheter placements approximately doubled. The rates of femoral and subclavian catheter placements decreased by approximately $50 \%$ and the rate of internal jugular catheter placement was roughly unchanged.

Figure 2 shows the numbers of femoral and nonfemoral catheter days by month for 2005 through 2007. The nonfemoral catheter days began to rise prior to the start of the hospitalist program and continued to rise afterward, showing an approximately 3 -fold increase by the end of the study period. The number of femoral catheters days was highly variable, but seemed to decrease by approximately $50 \%$.

Figure 3 shows the rates of femoral and nonfemoral catheter-associated bacteremia by month for 2005 through 2007 . The absolute number of infections in both periods was low and is shown at the top of each bar in the figure.

To our knowledge, there were no episodes of pneumothorax or major bleeding with PICC placement. There were 3 inadvertent arterial punctures, each of which was easily 


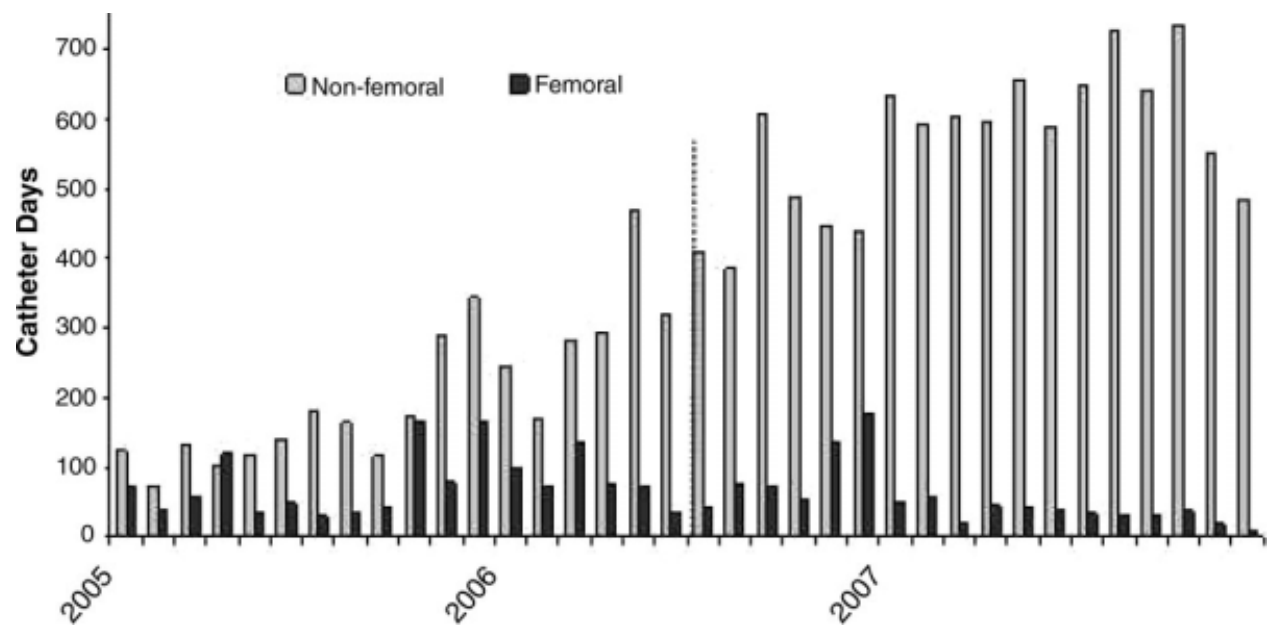

FIGURE 2. Femoral and nonfemoral catheter days by month. The dotted vertical line signifies the beginning of the hospitalist program.

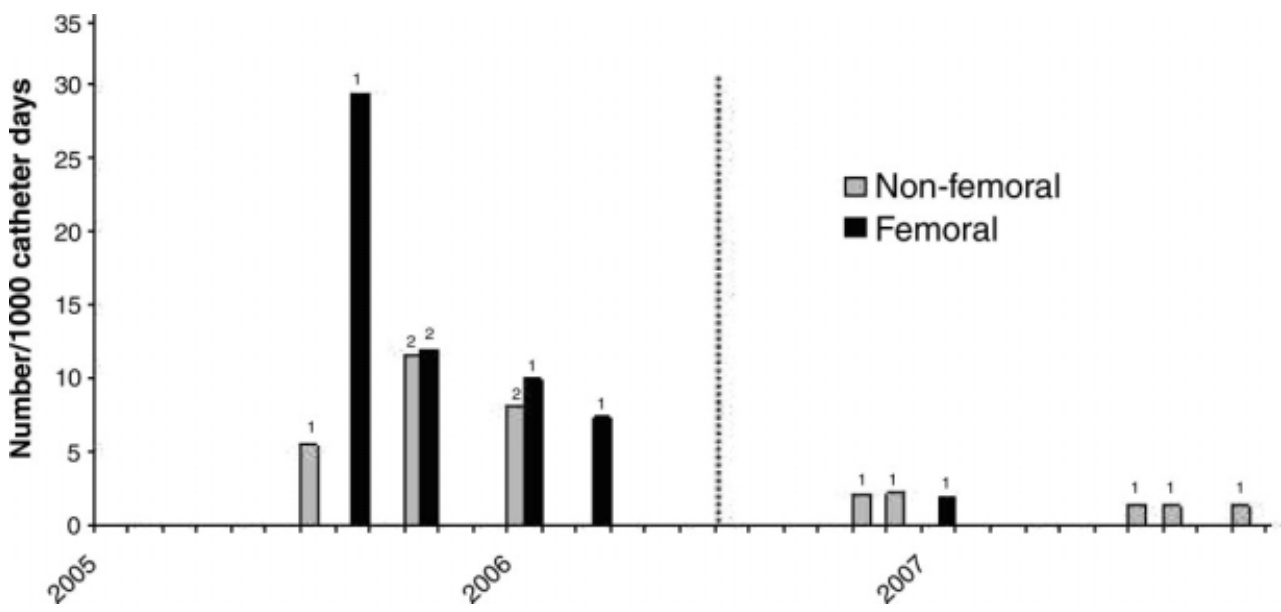

FIGURE 3. Femoral and nonfemoral bacteremia rates per 1000 catheter days by month. The dotted vertical line signifies the beginning of the hospitalist program. The absolute number of infections is noted atop each bar.

controlled with local pressure. There was 1 incident of a coiled guidewire that could not be removed at the bedside and had to be removed in interventional radiology with no significant consequence to the patient.

\section{Discussion}

The complications associated with central catheter insertion continue to place the hospitalized patient at risk. PICCs may offer significant advantages over other types of central catheters in terms of decreased rates of mechanical and infectious complications. Despite this, hospital physicians have not traditionally been trained to place PICCs. We have shown in our small, university-affiliated community hospital that training hospital physicians to place PICCs was associated with a decrease in the placement of centrally-inserted venous catheters and a reduced rate of femoral catheter days. At the same time, the rate of central catheter-related bacteremia remained low.
There are many limitations to our study. Since the analysis was retrospective and uncontrolled, it is not possible to attribute the decrease in femoral catheter days and the low infection rates solely to the use of PICCs. There may have been other factors, either related or unrelated to the transition to a hospitalist service, that influenced the results, such as improved hand hygiene, attention to the use of 5 barrier precautions, and the use of chlorhexidine cleansing. Also, since the study was descriptive and outcome measures were either not available or the numbers small, we cannot prove that there was benefit to the patients or that the changes in rates were statistically significant.

Training hospital physicians to place PICCs in our study was associated with a 2-fold increase in the overall rate of catheter placements. The reason for this increase in the total number of catheter placements is not clear, but it is likely related to the ease of PICC placement and the increasing number of patients with difficult intravenous access. It 
is unclear if an equivalent number of traditional central catheters would have been placed were the hospitalists not trained in PICC placement. However, this increase in total number of catheters did not appear to result in an increase in catheter-related bacteremia or in mechanical complications.

We observed no apparent decrease in the insertion rate of internal jugular catheters in our study, despite a decrease in the rates of subclavian and femoral catheter placements. Although the current CDC guideline recommends using the subclavian vein as the preferred site, the UK National Institute for Clinical Excellence (NICE) is now recommending the use of real-time ultrasound with each placement, ${ }^{18}$ and we find that this is best done in the internal jugular vein. Also, the rate of placement of femoral catheters remained higher than that of subclavian catheters-most likely because the femoral vein remained the site of choice for emergently-placed catheters - as PICC, more so than subclavian, became the preferred site for elective catheters.

Training physicians to place PICCs was not a simple task. In our experience, the availability of trainers at the tertiary care hospital was limited and the distractions of other duties of the hospitalist complicated the learning process. Two of our 5 physicians could not schedule time with the training nurse and were not able to acquire the skill. However, after training, the 3 hospitalists found that there was such a demand for PICCs that with time it was easy to maintain and even refine this skill. Since we only had 3 of 5 hospitalists trained in PICC placement, we could not have a PICC-trained hospitalist on site 24 hours a day and the remaining 2 physicians had to rely on centrally-placed catheters for access or have 1 of the trained physicians come to the hospital from home.

In summary, PICCs may be a safe and easy alternative to centrally-placed catheters for the hospital physician attempting to secure central intravenous access and may lead to a decrease in the need for more risky CVC insertions. More definitive, controlled investigation, with patient outcome data, will be required before this can be advocated as a universal recommendation.

\section{Address for correspondence and reprint requests}

Adam S. Akers, Assistant Professor of Critical Care Medicine, Chief, Division of Hospital Medicine, Department of Critical Care Medicine, University of Pittsburgh Medical Center, 611 Scaife Hall, 3550 Terrace Street, Pittsburgh, PA 15261; Telephone: 412-6470672; Fax: 412-647-8060; E-mail: akersas@upmc.edu Received 26 March 2008; revision received 16 September 2008; accepted 28 September 2008.

\section{References}

1. Climo M, Diekema D, Warren DK, et al. Prevalence of the use of central venous access devices within and outside of the intensive care unit: results of a survey among hospitals in the prevention epicenter program of the Centers for Disease Control and Prevention. Infect Control Hosp Epidemiol. 2003;24:942-945.

2. Kyle KS, Myers JS. Peripherally inserted central catheters: development of a hospital-based program. J Intraven Nurs. 1990;13:287-290.

3. Graham DR, Keldermans MM, Klem LW, et al. Infectious complications among patients receiving home intravenous therapy with peripheral, central, or peripherally placed central venous catheters. Am J Med. 1991; 91:95S-100S.

4. Skiest DJ, Abbott M, Keiser P. Peripherally inserted central catheters in patients with AIDS are associated with a low infection rate. Clin Infect Dis. 2000;30:949-952.

5. Lam S, Scannell R, Roessler D, Smith MA. Peripherally inserted central venous catheters in an acute care hospital. J Intraven Nurs. 1990;154: 1833-1837.

6. Black IH, Blosse SA, Murray WB. Central venous pressure measurements: peripherally inserted catheters versus centrally inserted catheters. Crit Care Med. 2000;28:3833-3836.

7. Thiagaragen R, Ramamoothry C, Gettman T, et al. Survey of the use of peripherally inserted central venous catheters in children. Pediatrics. 1997;99:e4.

8. Casalmir EC. Peripherally inserted central catheter (PICC) is effective in the care of critically ill patients using the basilic and cephalic veins and performed under ultrasound guidance at the patient's bedside by a pulmonary and critical care specialist. [October 23-28, 2004, Seattle, Washington, USA. Abstracts]. Chest. 2004;126(4 suppl):705S-1014S.

9. Williams JF, Seneff MG, Friedman BC, et al. Use of femoral venous catheters in critically ill adults: prospective study. Crit Care Med. 1991;19:550-553.

10. Mansfield PF, Hohn DC, Fornage BD, Gregurich MA, Ota DM. Complications and failures of subclavian-vein catheterization. N Engl J Med. 1994; 331:1735-1738.

11. Safdar N, Maki D. Risk of catheter-related bloodstream infection with peripherally inserted central venous catheters used in hospitalized patients. Chest. 2005;128:489-495.

12. Loewenthal MR, Dobson PM. The peripherally inserted central catheter (PICC): a prospective study of its natural history after cubital fossa insertion. Anaesth Intensive Care. 2002;30:21-24.

13. Chemaly RF, de Parres JB, Rehm SJ. Venous thrombosis associated with peripherally inserted central catheters: a retrospective analysis of the Cleveland Clinic experience. Clin Infect Dis. 2002;34:1179-1183.

14. Ong B, Gibbs H, Catchpole I, Hetherington R, Harper J. Peripherally inserted central catheters and upper extremity deep vein thrombosis. Australas Radiol. 2006;50:451-454.

15. Abdullah BJ, Mohammad N, Sangkar JV, et al. Incidence of upper limb venous thrombosis associated with peripherally inserted central catheters (PICC). Br J Radiol. 2005;78:596-600.

16. Pradoni P, Polistena P, Benardi E, et al. Upper-extremity deep vein thrombosis: risk factors, diagnosis, and complications. Arch Intern Med. 1997; 157:57-62.

17. Fong NI, Holtzman SR, Bettmann MA, Bettis SJ. Peripherally inserted central catheters: outcome as a function of the operator. $J$ Vasc Interv Radiol. 2001;12:723-729.

18. Hind D, Calvert N, McWilliams R, et al. Ultrasonic locating devices for central venous cannulation: meta-analysis. BMJ. 2003;327:361. 